\title{
Evaluation Reference and Error Function Unification for Shape Error
}

\author{
Xianping $\mathrm{Tu}^{1, \mathrm{a}}$, Xianqing Lei ${ }^{1, \mathrm{~b}^{*}}$ \\ ${ }^{1}$ Henan University of Science \& Technology Luoyang China \\ aemail:xptu2000@126.com, bemail:ly-lxq@163.com
}

Key words: Shape error, Evaluation reference, Error function, Unification

Abstract. According to definitions, geometric characteristics and corresponding error evaluation functions of straightness error, flatness error, roundness error, cylindricity error and sphericity error, the concept of evaluation reference of form error is put forward by analyzing the minimum zone evaluation method of form error. The evaluation reference corresponding to form error is obtained by studying the function of all kinds of form error. The relationship between the evaluation references and the all kinds of form error are studied. The unified formula of the form error is gained and the unification of the form error is realized by summing up the relationships. The foundation of researching the unified shape error evaluation algorithm is laid by unified mathematical description.

\section{Introduction}

In the process of mechanical manufacturing, due to some geometric errors of machine tool, fixture, cutting tool, work-piece system, and the influence of forced deformation, thermal deformation, vibration, tool wear and internal stress changes in work material, shape error will be produced. The working precision, connection strength, motion stability, sealing, wear resistance, noise and service life of mechanical products will be influenced by shape error. So the study of the shape error evaluation method in high-precision has great significance for the work performance of whole machine.

Evaluation of shape error is a necessary method for judging whether the shape of the measured geometric elements meet the design demand of shape precision. The least square method and minimum zone method are the primary method for assessing shape error. Least squares shape error is obtained in the condition that the quadratic sum of the distance between measured points and desired shape is minimum. Because of its simple principle and convenient operation, the proposed method has been used in many measuring instrument for shape error. But the basic principle doesn't apply to the minimum condition principle of shape error, so the method can not be used in the shape error evaluation of high-precision parts because of the principle error. The minimum zone which containing the measured shape (width, diameter, radius difference) is constituted by the minimum conditions. The shape error value is simple and direct-viewing by using the minimum zone method. Therefore, the minimum zone evaluation method is stipulated by the national standards and ISO standards as the arbitration method for shape error evaluation.

Calculating the shape error is solving the optimal value of nonlinear function, because shape error functions are all nonlinear. Calculating the shape error is maxima minimization or minima maximization problem in math which is resolved by optimizing solution according to the objective function of measured object. Currently, many research results have been achieved by shape error evaluation in evaluation algorithms of single form error[1-6]. With the extensive research of the evaluation method, theory, calculation methods and other issues of shape error, the unified algorithm system of shape error evaluation was tentative studied by some scholars. Based on natural selection and genetic mechanism, the evolutionary algorithm of shape error is proposed by Liu [7], through simulating search and optimization algorithms of biology evolution mechanism, the search space (solution space) was mapped to genetic space. $\mathrm{Su}[8]$ establish a unified saddle point programming model for shape error assessment and use the genetic algorithm to directly calculate. The measurement results of shape error are deduced, which approximately obey the normal distribution, and the result was verificated by experiment. The shape error evaluation is unified into solving the problem that making the maximum deviation of measurement point vector 
and control plan vector to minimum by Yue[9]. The unification of the form error evaluation is realized by using quadratic programming successive approximation. Sequence converges to the unknown optimal solution is generated. These researching results have an important role in promoting the research of theory and method of shape error evaluation unification.

According to the definition, principle and geometrical characteristics of different shape errors, the corresponding evaluation methods are studied and analyzed. The definition of shape error evaluation reference is proposed. Evaluation reference of the appropriate shape error is defined. Unified shape error function based on the evaluation of the minimum zone condition is obtained. Through the transformation parameters to meet the need of different shape error evaluation, the function unification of the shape error is realized. The foundation of researching the unified shape error evaluation algorithm is laid by unified mathematical description.

\section{The evaluating reference of shape error}

The standard is constituted by one or more elements that relations with the measured elements and used to determine geometric location relation on the ideal geometric elements (point, line, or surface). According to the "Minimum Condition" concept of shape error, the determination of ideal geometry position is the core of minimum zone evaluation of shape error in evaluating shape error. The ideal geometrical orientation is depend on the ideal geometrical reference orientation. When the cylindricity error is evaluated, the ideal geometry shape is an ideal cylindrical surface with a certain spatial orientation. The orientation of ideal cylindrical surface is depend on the orientation of ideal cylindrical axis. The cylindricity error is depend on ideal cylindrical surface . The radius difference between two coaxial cylindrical surfaces including measured points has no relationship with the radius of the cylindrical surface, it only has relationship with the spatial orientation of the ideal cylindrical surface,that is it only has relationship with the ideal orientation of the cylindrical axis. Therefore, the axis of ideal cylindrical surface is defined as the evaluation reference for cylindricity error by author. The problem of looking for spatial orientation of ideal cylindrical surface is transformed into the problem of looking for spatial orientation of ideal cylindrical surface axis. According to the definition of form error, the function of shape error is the distance between measurement point and reference point, measurement point and base line.

\section{Function Unification of Shape Error}

If the measured object is different, the ideal geometric shape and the evaluation reference of shape error are also different. The main evaluation reference of shape error is point (roundness, sphericity and so on) and the straight line (straightness, cylindricity, flatness and so on). Calculating the distance extreme difference of measuring points and reference point, measurement points and base line is the essential issue for shape error evaluation. So, the function of shape error evaluation is denoted by the distance formula of measurement points and reference point, measurement points and base line.

If measurement points are denoted by $P_{i}\left(x_{i}, y_{i}, z_{i}\right)(i=1,2, \ldots, N)$, reference point and points on the base line are denoted by $O\left(x_{0}, y_{0}, z_{0}\right)$, the direction numbers of base line are denoted by $l, m, n$, the unified formula of shape error function is:

$$
D_{i}=\sqrt{\left[\left(x_{i}-x_{0}\right)^{2}+\left(y_{i}-y_{0}\right)^{2}+\left(z_{i}-z_{0}\right)^{2}\right] \cdot k_{1}-\frac{\left[l\left(x_{i}-x_{0}\right)+m\left(y_{i}-y_{0}\right)+n\left(z_{i}-z_{0}\right)\right]^{2}}{l^{2}+m^{2}+n^{2}} \cdot k_{2}} .
$$

The shape error is :

$\Delta D=\max \left(D_{i}\right)-\min \left(D_{i}\right)$.

In formula (1), $k_{1}, k_{2}$ are the function coefficients of shape error . 


\section{The application of the unified function of error}

When the measured object is plane linear, the measured points are expressed by $P_{i}\left(x_{i}, y_{i}\right)(i=1,2, \ldots, N)$, the equation of base line is denoted by $l x_{0}+m y_{0}+d=0$.In the Eq.(1), $k_{1}=0, k_{2}=-1, n=0, z_{0}=0$.The Eq.(1) becomes distance formula of measurement point and linear in the plane, the error function of plane straightness is obtained.

$$
D_{i}=\sqrt{\frac{\left[l\left(x_{i}-x_{0}\right)+m\left(y_{i}-y_{0}\right)\right]^{2}}{l^{2}+m^{2}}}=\frac{\left|l\left(x_{i}-x_{0}\right)+m\left(y_{i}-y_{0}\right)\right|}{\sqrt{l^{2}+m^{2}}}
$$

(3)

In formula (3), $\left(x_{0}, y_{0}\right)$ is the point in the base line, $l, m$ is direction number of base line.

Minimum zone error of plane straightness error is the minimum distance between two parallel lines containing all measurement points in the plane. That is, $\Delta D=\min \left(D_{i}\right)$.

When the measured object is space linear, the measured points are expressed by $P_{i}\left(x_{i}, y_{i}, z_{i}\right)(i=1,2, \ldots, N)$, the equation of base line is denoted by $\left(x-x_{0}\right) / l=\left(y-y_{0}\right) / m=\left(z-z_{0}\right) / n$. In the Eq.(1), $k_{1}=0, k_{2}=-1$. The Eq.(1) becomes distance formula of measurement points and linear in the space, the error function of space straightness error is obtained.

$$
D_{i}=\sqrt{\frac{\left[l\left(x_{i}-x_{0}\right)+m\left(y_{i}-y_{0}\right)+n\left(z_{i}-z_{0}\right)\right]^{2}}{l^{2}+m^{2}+n^{2}}}=\frac{\left|l\left(x_{i}-x_{0}\right)+m\left(y_{i}-y_{0}\right)+n\left(z_{i}-z_{0}\right)\right|}{\sqrt{l^{2}+m^{2}+n^{2}}} .
$$

(4)

In formula (4), $\left(x_{0}, y_{0}, z_{0}\right)$ is the point in the base line, $l, m, n$ are direction number of base line.

Minimum zone error of space straightness is the smallest diameter of spatial cylinder containing all measurement points. That is, $\Delta D=2 \min \left(D_{i}\right)$.

When the measured object is space plan, the measured points are expressed by $P_{i}\left(x_{i}, y_{i}, z_{i}\right)(i=1,2, \ldots, N)$, the equation of base plane is denoted by $l x_{0}+m y_{0}+n z_{0}+d=0$. In the Eq.(1) $k_{1}=0, k_{2}=-1$, The Eq.(1) becomes distance formula of measurement points and line(normal of base plane) in the space. The error function of space flatness error is obtained.

$$
D_{i}=\sqrt{\frac{\left[l\left(x_{i}-x_{0}\right)+m\left(y_{i}-y_{0}\right)+n\left(z_{i}-z_{0}\right)\right]^{2}}{l^{2}+m^{2}+n^{2}}}=\frac{\left|l\left(x_{i}-x_{0}\right)+m\left(y_{i}-y_{0}\right)+n\left(z_{i}-z_{0}\right)\right|}{\sqrt{l^{2}+m^{2}+n^{2}}} .
$$

In formula (5), $\left(x_{0}, y_{0}, z_{0}\right)$ is the point on the base plane, $l, m, n$ are direction number of the base plane.

Minimum zone error of space flatness error is the minimum distance between two parallel planes in the space containing all measurement points. That is, $\Delta D=\max \left(D_{i}\right)-\min \left(D_{i}\right)$.

When the measured object is cylindricity, the measured points are expressed by $P_{i}\left(x_{i}, y_{i}, z_{i}\right)(i=1,2, \ldots, N)$, the base line of cylindricity error evaluation(space straight line) is denoted by $\left(x-x_{0}\right) / l=\left(y-y_{0}\right) / m=\left(z-z_{0}\right) / n$. In the Eq.(1), $k_{1}=0, k_{2}=-1$. The Eq.(1) becomes distance formula of measurement points and linear in space. The error function of cylindricity error is obtained.

$$
D_{i}=\sqrt{\frac{\left[l\left(x_{i}-x_{0}\right)+m\left(y_{i}-y_{0}\right)+n\left(z_{i}-z_{0}\right)\right]^{2}}{l^{2}+m^{2}+n^{2}}}=\frac{\left|l\left(x_{i}-x_{0}\right)+m\left(y_{i}-y_{0}\right)+n\left(z_{i}-z_{0}\right)\right|}{\sqrt{l^{2}+m^{2}+n^{2}}} .
$$

(6)

In formula (6), $\left(x_{0}, y_{0}, z_{0}\right)$ is the point in the reference line, $l, m, n$ are the direction number of 
the reference line which across the point $\left(x_{0}, y_{0}, z_{0}\right)$.

Minimum zone error of cylindricity error is the minimum radius difference between two coaxial cylindrical surface containing all measurement points. That is, $\Delta D=\max \left(D_{i}\right)-\min \left(D_{i}\right)$.

When the measured object is circular curve on the plane, the measured points are expressed by $P_{i}\left(x_{i}, y_{i}\right)(i=1,2, \ldots, N)$. In Eq.(1), $k_{1}=1, k_{2}=0, z_{i}=0, z_{0}=0$, the Eq.(1) becomes a formula of measurement points and the reference points. The error function of roundness error is obtained.

$$
D_{i}=\sqrt{\left(x_{i}-x_{0}\right)^{2}+\left(y_{i}-y_{0}\right)^{2}} \text {. }
$$

(7)

In formula (7), $\left(x_{0}, y_{0}\right)$ is the reference point of the measured circular curve.

Minimum zone error of roundness error is the minimum radius difference of the two concentric circles containing all measurement points. That is, $\Delta D=\max \left(D_{i}\right)-\min \left(D_{i}\right)$.

When the measured object is sphericity, the measured points are expressed by $P_{i}\left(x_{i}, y_{i}, z_{i}\right)(i=1,2, \ldots, N)$. In Eq.(1), $k_{1}=1, k_{2}=0$. The Eq.(1) becomes a formula of measurement points and the reference points. The error function of sphericity error is obtained.

$$
D_{i}=\sqrt{\left(x_{i}-x_{0}\right)^{2}+\left(y_{i}-y_{0}\right)^{2}+\left(z_{i}-z_{0}\right)^{2}} \text {. }
$$

$(8)$

In formula (8), $\left(x_{0}, y_{0}, z_{0}\right)$ is the reference point of the measured spherical surface.

Minimum zone error of the measured spherical surface is the minimum radius difference of the two concentric sphere surfaces containing all measurement points. That is, $\Delta D=\max \left(D_{i}\right)-\min \left(D_{i}\right)$.

\section{Conclusions}

Based on the definition of the shape error and the shape error evaluation criterion of minimum condition, the concept of form error evaluation benchmark is put forward and the uniform mathematical description of shape error function is achieved. Error functions of different shape errors can be got by change the coefficient of the unified function of shape error. The foundation of unified evaluation algorithm of form error is laid by this study.

\section{Acknowledgments}

The authors gratefully acknowledge Basic and Frontier Technology research proposal of Henan Province ( No.122300410114 for financial support of this research work.

\section{References}

[1] Y. Hou and J. Lv: Chinese Journal of Scientific Instrument, Vol. 17 (1996) No.6, pp.618622.(in Chinese)

[2] Z. Long: Research on Model and Algorithm of Shape Error Evaluation in Three Coordinate Measuring System (MS.,Zhongshan University,China 2008), pp.1-79.(in Chinese)

[3] N.Y. Dai: Research on Error Evaluation Method for Complex Geometry Shape (MS.,Central South University,China 2010), pp.1-73.(in Chinese)

[4] J.N. Zhang: Tolerance Modeling and Error Evaluation Techniques for Shape Based on GPS Standard System (MS.,Zhejiang University,China,2007), pp.1-77.(in Chinese)

[5] P.B. Danish and M.S. Shunmugam: Computer Methods in Applied Mechanics and Engineering, Vol. 92 (1991) NO.3, pp.309-324. 
[6] P. Liao: Study on Calculation of Form Error Based on Genetic Algorithm (Ph.D.Central South University,China 2002), pp.1-123.(in Chinese)

[7] Y.C. Liu and M. Chen: Acta Metrologica Sinica, Vol. 22 (2001) No.1, pp.18-22.(in Chinese)

[8] S.P. Li, S.Y. Li and G.L. Wang: Acta Metrologica Sinica, Vol. 24 (2003) No.1, pp.26-28.(in Chinese)

[9] W.L. Yue and Y. Wu: Journal of Agricultural Machinery, Vol.38 (2007) No.12, pp.169-172.(in Chinese) 